\title{
Two-phase MRI brain tumor segmentation using Random Forests and Level Set Methods
}

\author{
László Lefkovits \\ Sapientia University \\ Department of Electrical Engineering \\ Romania, Tg. Mureș \\ lefkolaci@ms.sapientia.ro
}

\author{
Szidónia Lefkovits \\ "Petru Maior" University \\ Department of Computer Science \\ Romania, Tg. Mureș \\ szidonia.lefkovits@science.upm.ro
}

\begin{abstract}
Magnetic resonance images (MRI) in various modalities contain valuable information usable in medical diagnosis. Accurate delimitation of the brain tumor and its internal tissue structures is very important for the evaluation of disease progression, for studying the effects of a chosen treatment strategy and for surgical planning as well. At the same time early detection of brain tumors and the determination of their nature have long been desirable in preventive medicine. The goal of this study is to develop an intelligent software tool for quick detection and accurate segmentation of brain tumors from MR images.

In this paper we describe the developed two-staged image segmentation framework. The first stage is a voxelwise classifier based on random forest (RF) algorithm. The second acquires the accurate boundaries by evolving active contours based on the level set method (LSM). The intelligent combination of two powerful segmentation algorithms ensures performances that cannot be achieved by either of these methods alone.

In our work we used the MRI database created for the BraTS '14-' 16 challenges, considered a gold standard in brain tumor segmentation. The segmentation results are compared with the winning state of the art methods presented at the Brain Tumor Segmentation Grand Challenge and Workshop (BratsTS).
\end{abstract}

\section{Keywords}

Brain tumor, multimodal MRI, voxel-wise segmentation, random forest, level set method, feature selection, tumor structure, hierarchical segmentation, supervised learning.

\section{INTRODUCTION}

Early detection of diseases is of the utmost importance to maintaining or somehow regaining one's health, and thus it contributes to improving quality of life. The combination of various image processing techniques creates an efficient diagnostic tool. One part of the imaging techniques is built around automatic image segmentation, which is much faster than time-consuming analysis by experts.

Cerebral metastases usually become symptomatic in the form of headaches, focal neurological deficits or seizures, but they may also be found coincidentally in cancer staging scans. In any case, the earlier the tumor is detected, the better the chances of survival. In addition to sensitive automatic detection, precise segmentation of tumors is also required for efficient treatment and intervention planning. In particular,

Permission to make digital or hard copies of all or part of this work for personal or classroom use is granted without fee provided that copies are not made or distributed for profit or commercial advantage and that copies bear this notice and the full citation on the first page. To copy otherwise, or republish, to post on servers or to redistribute to lists, requires prior specific permission and/or a fee. brain tumor segmentation consists of separating the different tumor tissues from normal brain tissue. Accurate and reproducible segmentation and characterization of abnormalities can be considered indispensable in medical diagnosis.

The subsequent sections of the paper are organized as follows: in section 2 the milestone approaches of the literature are summarized. In section 3 the first major stage of the proposed system, the random forest (RF), is described, followed by the mathematical details of the second stage, the level set method (LSM), in section 4. Finally, the results of our experiment (section 5) are presented with an emphasis on the improvement brought by the LSM. The performances obtained are compared to other systems and conclusions are drawn.

\section{RELATED WORK}

At present, there are many state-of-the-art brain tumor segmentation methods that have been developed. These have been implemented and published mainly for the Brain Tumor Image Segmentation Benchmark, organized yearly since 2012 [1]. There are two main categories: generative and discriminative models. Generative methods attempt to determine the probability distribution 
function between the input and the target outputs. They rely on the Bayes theorem and are based on prior knowledge using appearance or anatomic properties. All these methods assume standardized data acquisition, registration and alignment in order to be converted into a generally usable probabilistic model [1]. On the other hand, discriminative models are capable of learning the classification function directly from a manually labeled training dataset. The main drawback is the requirement for a substantial amount of data in order to create sufficiently general and high-performing classifiers via supervised learning.

Today's leading architectures in the field of medical image processing and brain tumor segmentation are based on two major methods: the random forest decision tree ensemble [3] and deep learning via convolutional neural networks (CNN) [4].

Zikic et al. [5] combine a discriminative model using 40 decision trees in the classification ensemble with 2000 context-aware attributes, combining all of these with a generative model using tissue-specific probabilities for each patient.

Ellwaa et al. [6] create a random decision tree with an iterative approach using heuristics to gradually add the data from new patients to the training dataset.

Maier et al. [7] use the random forest classifier for the prediction of ischemic stroke lesion outcome. They include texture as anatomical features in the 200-tree ensemble.

Another radically different classification and segmentation approach is based on a state-of-the-art method called Deep Learning.

Chang proposed in [4] a very fast but highly accurate CNN architecture with few parameters. In this classification, the deepest convolutional output layers are combined with hyperlocal features from the input image.

Soltaninejad et al. [8] join the two methods. They utilized the VGG16 [9] fully convolutional neural net to obtain a feature map that is combined with a Gabor filter bank. All of these feature maps are fed to a random forest classifier.

The Level Set Method (LSM) proposed by ChanVese [10] is used to determine the active contour between two surfaces by minimizing the sum of intensity variance of the defined inner and outer regions. It is used for medical image segmentation only in combination with other segmentation methods [11, 12].

\section{RANDOM FOREST}

The random forest (RF) is an ensemble of decision trees suitable for the task of classification. It is one of the few methods applicable for a very large dataset, for example 3D medical images. Beside classification, it can also be used for feature selection because it estimates variable importance during the steps of the algorithm. The multitude of randomly generated decision trees representing the forest has very good generalization properties owing to the randomization process used in the construction of each tree. Each of the trees represents a unique weak classifier. The ensemble joins several such trees, thereby obtaining a strong classifier. The underlying database is randomly sampled with replacement and, for each tree, a different bootstrap set and out-of-bag (OOB) set is obtained. The bootstrap set is used in the creation of the tree. The OOB set (disjunctive to the bootstrap set) is used for evaluation purposes, for the computation of the generalized error of the ensemble. Not only are the data instances used randomized in each tree, but the splitting criterion of a tree-node is also based on randomness. Out of a large number $(M)$ of variables (features) only a given number $\left(m_{\text {tires }}<<M\right)$ are selected randomly for splitting. The optimum of the splitting criterion is computed only for these selected variables, based on the maximization of information gain. The OOB error is computed for each tree on the OOB set, using the tree structure obtained. The average OOB error of the ensemble is the unbiased estimator of the generalized error of the model (GE). [13]

The minimization of the generalized error involves the optimization of the RF parameters. The parameters which have to be tuned in order to obtain a well-working classifier are the number of trees in the ensemble $\left(K_{\text {trees }}\right)$, the number of nodes in each tree $\left(T_{\text {nodes }}\right)$ and the number of variables used as a splitting criterion in the nodes, called number of tries $\left(m_{\text {tries }}\right)$. The number of trees $\left(K_{\text {trees }}\right)$ influence the generalization error of the ensemble. If it is sufficiently large, the overfit of classification can be avoided, but the generalization error grows and the computation time increases. The number of nodes $\left(T_{\text {nodes }}\right)$ is usually not limited in many of the other attempts in the literature. We have discovered that limitation is very important in order to avoid extremely deep trees. The third parameter is the number of variables $\left(m_{\text {tries }}\right)$ randomly selected in each node. This value restricts the variables evaluated for finding the optimal split.

In our segmentation approach we make use of both the classification capacity of the RF ensemble and its variable importance measures applied in feature selection. The first step of creating the model is to fix a large number of low-level features (first order operators [mean, standard deviation, min, max, median, gradient]; higher order operators [difference of Gaussian, Laplacian, entropy, curvatures, kurtosis, skewness]; texture features [Gabor wavelets]; spatial context features [symmetry, projections, 
neighborhoods]), out of which the random forest is able to choose the most important ones. Only after this step does the training of the RF classifier described above follow, using the important features only. In statistical pattern recognition, the more adequate features are selected, the better the final decision will be. The RF approach offers an opportune method for the selection of relevant variables. In the case of RF, there are two possibilities to evaluate variable importance: Gini importance and permuted importance [13]. The variable importance depends on the RF ensemble obtained. Because the ensemble is based on randomness, the effective values of the importance are different for each new RF, but the order of important variables is, on average, similar. In our previous article we proposed a feature selection approach using the variable importance given by RF. Due to this algorithm, we managed to considerably reduce the number of initial variables $(V)$ to a much smaller amount $\left(V_{i m p}<V\right)$, which are considered important with regard to brain tumor segmentation. The algorithm proposed consists of the following steps:

1. Create an RF ensemble for variable importance evaluation;

2. Considering the order of importance, eliminate the least important $p \%$ of variables.

3. If variables are sufficiently reduced, continue with step 4, otherwise repeat from step 1.

4. Create the RF classifier considering the remaining variables.

5. Evaluate the classification performances obtained.

6. Accept or reconsider the number of iterations (steps 1-3) based on the classification accuracy.

In our experiments we considered different values of $p \%$ and a different number of iterations. At first, we were able to reduce a large number of unimportant variables, but in the last stages, only a few. This depends on the classification performances of the RF ensemble obtained.

\section{LEVEL SET METHOD}

The accurate segmentation of MR images is a difficult task due to unclear or blurred dividing surfaces between tissues. The level set method is used with predilection because it performs better than other segmentation algorithms such as the gradient, threshold or clustering methods. The performances are explained by the fact that in the level set method, the global proprieties of image intensities matter more than local ones. The variant of the level set method try to find an active contour which delimitates the image regions and evolves in time during the segmentation process. For this task we adopted the Chan-Vese algorithm [10], which tries to find the active contour by energy minimization. Namely, the sum of the intensity variance of segmented regions is minimized. Thus, the best location of the contour is in the force equilibrium state in the force field of the image. Furthermore, the implicit formulation of the active contour provides certain remarkable features, such as topological flexibility, good numerical stability and straightforward extension of the $2 \mathrm{D}$ formulation to the n-dimension.

The segmentation task can be enunciated by finding a curve $(C)$ that separates the image $(\Omega)$ into disjointed regions $\left(\Omega_{l}, \Omega_{2}, \ldots, \Omega_{n}\right)$. Mathematically, this can be formulated to find the curve $(C)$ which minimizes the Mumford-Shah functional:

$$
\begin{aligned}
& F\left(c_{1}, c_{2}, C\right)=\mu L(C)+v A(\operatorname{in}(C))+ \\
& +\lambda_{1} \int_{\text {in }(C)}\left|u_{0}(x, y)-c_{1}\right|^{2} d x d y+ \\
& +\lambda_{2} \int_{\text {out }(C)}\left|u_{0}(x, y)-c_{2}\right|^{2} d x d y
\end{aligned}
$$

where $c_{1}$ and $c_{2}$ are the average intensity levels inside and outside of the contour, $L(C)$ is the length of curve, $A(i n(C))$ the area inside the curve, $u_{0}(x, y)$ image intensities and the $\mu, v, \lambda_{1}, \lambda_{2}$, parameters should be determined for each segmentation type.

In the level set formulation, instead of searching for the solution in terms of $C$, we are looking for a surface $\Phi(x, y)$ with the following properties:

$$
\begin{aligned}
& C=\{(x, y) \in \Omega: \Phi(x, y)=0\} \\
& \text { inside }(C)=\{(x, y) \in \Omega: \Phi(x, y)>0\} \\
& \text { outside }(C)=\{(x, y) \in \Omega: \Phi(x, y)<0\}
\end{aligned}
$$

where $\Phi(x, y)$ is the signed distance function from $C, O$ on curve $C$, negative outside $\Phi$ and positive inside $\Phi$. The distance function $\Phi(x, y)$ evolves in time in such way that the curve $C$ is the zero-level set of $\Phi(x, y, t)$

$$
\begin{aligned}
& F\left(c_{1}, c_{2}, C\right)= \\
& =\mu \int_{\Phi} \delta_{0}(\Phi(x, y))|\nabla \Phi(x, y)| d x d y+ \\
& +v \int_{\Phi} H(\Phi(x, y)) d x d y+ \\
& +\lambda_{1} \int_{\Omega}\left|u_{0}(x, y)-c_{1}\right|^{2} H(\Phi(x, y)) d x d y+ \\
& +\lambda_{2} \int_{\Omega}\left|u_{0}(x, y)-c_{2}\right|^{2} H(1-\Phi(x, y)) d x d y
\end{aligned}
$$


where $\delta$ is the Dirac function and $H$ is the Heaviside function determining the inside (outside) of curve $C$. The first term is the length of the curve, the second is the area inside the curve, the third and fourth terms are energy terms inside and respectively outside the curve. Using the level set formulation, the image segmentation becomes an energy minimization problem, which leads to the solution with the corresponding Euler-Lagrange equation:

$$
\frac{\partial \Phi}{\partial t}=\frac{\partial F}{\partial \Phi}
$$

By using the Gateaux derivate of the energy function $\partial \mathrm{F} / \partial \Phi$ we obtain the corresponding Euler-Lagrange equation:

$$
\begin{aligned}
& \frac{\partial \Phi}{\partial t}=\delta(\Phi)[\mu \kappa(\Phi)|\nabla \Phi|-v- \\
& \left.\lambda_{1}\left(u_{0}-c_{1}\right)^{2}+\lambda_{2}\left(u_{0}-c_{2}\right)^{2}\right]
\end{aligned}
$$

where $\kappa(\Phi)$ is the curvature of $\Phi, u_{0}(x, y)$ image intensities and the $\mu, v, \lambda 1, \lambda 2$, parameters should be determined for each segmentation type.. This partial derivate equation (PDE) can be easily solved with the standard gradient descent using variational methods. In this framework, the $c_{1}$ and $c_{2}$ are constant in the inside and outside region, respectively, and can be determined by

$$
\begin{gathered}
c_{1}(\varphi)=\frac{\int_{\Omega} u_{0}(x, y) H(\varphi(x, y)) d x d y}{\int_{\Omega} H(\varphi(x, y)) d x d y} \\
c_{2}(\varphi)=\frac{\int_{\Omega} u_{0}(x, y)(1-H(\varphi(x, y))) d x d y}{\int_{\Omega}(1-H(\varphi(x, y))) d x d y}
\end{gathered}
$$

The $c_{1}$ and $c_{2}$ are the mean values of intensities in the segmented regions, inside and outside the curve $C$, respectively. It is desirable for these regions to be as homogeneous as possible. Taking this into account, we have to compute the level set function not on the whole image domain, but only in a narrow band near the different tumor tissue contours. This way, we managed to exploit the advantage of precise delimitation and at the same time reduce computation time.

\section{RESULTS AND EXPERIMENTS}

The primary task of segmentation is the delimitation of the tumor tissue from healthy brain tissue. At the same time, we also propose to determine the tumor structure by considering only four specific tissue types: the edema as well as three tumor substructures, which are the non-enhancing (solid) core, the enhancing tumor core and the necrotic (or fluidfilled) core [1]. These structures offer much more visual information for radiologists than a biological interpretation.

Our experimental setup utilizes the image database created for purposes of evaluating the approaches implemented participating in the BraTS Challenges ("12-"17) [2]. This database has become a gold standard in brain tumor segmentation during the last six years. The images were acquired in highly reputable clinic centers with different $1.5 \mathrm{~T}$ or $3 \mathrm{~T}$ MRI equipment, but strictly based on a standardized acquisition protocol. Experts in the field manually annotated the images using a segmentation protocol described in [14]. The manual annotation and segmentation of MR images is very time-consuming and requires fastidious and careful work even from an experimented specialist.

Each image set in the database consists of five types of registered images: T1, T1c (with the contrast material Gadolinium), T2, FLAIR and the expertannotated image. Furthermore, the annotation contains four tumor classes: edema, enhanced tumor, non-enhanced tumor and necrotic core. The SICAS medical image repository [2] offers more than a hundred test image sets for evaluation, giving numerical performance results without showing the annotated image. In this online evaluation system there are only three classes which are taken into account and considered representative in clinical practice: Whole Tumor - WT (including all four tumor tissues), Tumor Core - TC (including all tumor structures except for edema) and Active Tumor - AT (only the enhancing core). The novelty of this article is the extension of our previous framework with a new stage in order to increase segmentation performances.

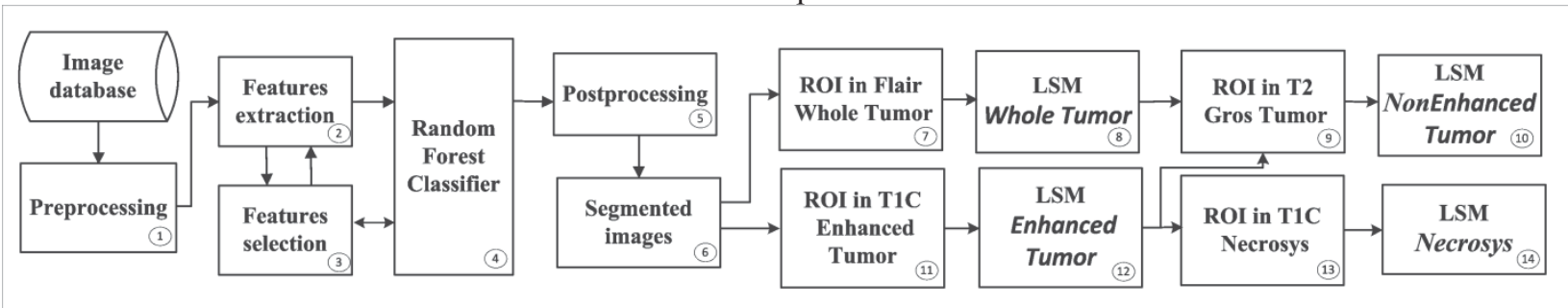

Figure 1. Block diagram of the proposed system 
The first stage of the framework proposed is a voxelwise segmentation based on the random forest (RF) algorithm and is described in detail in our previous work [15]. The first stage corresponds to the blocks (1)-(6) in Figure 1.

The delimitation surface between tissues approximates the gold standard only roughly, and the internal tumor structure detected differs slightly from the annotation. In order to improve the segmentation results obtained after the random forest approach, our idea is to refine the contour of tumor tissues by applying the level set method. This method has two major drawbacks: it requires adequate initialization and is only capable of delimit nearly homogenous regions. The first drawback is overcome by considering the initial curve provided by the previous segmentation stage obtained from the RF approach. Secondly, we propose to determine the internal structure of the tumor in multiple steps starting from the inside towards the outside of the tumor. This layered detection of the different tumor tissues corresponds to the expect annotation protocol described in [14].

The primary assumptions of accurate medical image processing are the images without artifacts or noise. In addition, well-defined and repeatable correspondence between tissues and pixel intensities is also expected. In order to fulfill the desired criteria we applied three important correction procedures, in the following order: bias-field correction, noise filtering and intensity standardization in preprocessing.

For voxel-wise segmentation we transformed the image database previously described into a numeric database where each instance corresponds to a voxel, and the attributes are the values of several local image features. The problem is to determine the most significant features for the segmentation task proposed. In this field there is no recipe; every author defines the feature set based on their own experience or intuition. We defined 240 low-level image features in each image modality (T1, T1C, T2, Flair) and obtained a 960-feature set $(V=4 \times 240)$ that characterizes a voxel and its surroundings. However, a single 3D image from the database used contains about 1.5 million pixels; in our setup, the training database contains 50 brain images occupying about 500 GB of memory. Such a large database is practically unmanageable, and therefore we need to reduce it.

There are two ways of reducing this size: reducing the number of instances and/or the number of features. The number of instances can be reduced by random subsampling of the database. The number of instances belonging to the healthy brain tissue-class is ten times larger than the instances belonging to the tumor-class, and thus a sampling of 10:1 does not cause loss of information.

After this sampling of instances the database still remains large, and therefore it is necessary to reduce the number of features as well. Using the algorithm we proposed for variable importance evaluation, we managed to select the 120 most important features $\left(V_{\text {imp }}\right)$ to be applied in this segmentation process. We showed that the OOB error obtained by the classifier build on this reduced feature set remains almost the same with the reduced set. The algorithm proposed in [15] uses the random forest variable importance evaluation and is able to run on the very large database.

The parameter optimization of the random forest and the methods applied for building a well-performing classifier for MR brain tumor segmentation is explained in our article [16]. Our optimized classifier is composed of $K_{\text {trees }}=100$ trees, each having a size of $T_{\text {nodes }}=2048$ nodes. The splitting criterion is evaluated with $m_{\text {tries }}=9$ randomly chosen features out of the whole $M=120$ features/voxel. The classification results obtained on the BraTS 2016 test set are given in (Table 1, column 3).

The results obtained are comparable with the latest reported results (Table 1, columns 1-2), described in [1].

\begin{tabular}{|c|c|c|c|c|}
\hline & $\begin{array}{c}\text { BraTS } \\
2012[1]\end{array}$ & $\begin{array}{c}\text { BraTS } \\
2013[1]\end{array}$ & $\begin{array}{c}\text { Our RF } \\
\text { classif. }\end{array}$ & $\begin{array}{c}\text { Our } \\
\text { 2staged } \\
\text { classif. }\end{array}$ \\
\hline WT & $\begin{array}{c}0.63- \\
0.78\end{array}$ & $\begin{array}{c}0.71- \\
0.87\end{array}$ & $\mathbf{0 . 7 5 - 0 . 8 6}$ & $\mathbf{0 . 8 0 - 0 . 9 1}$ \\
\hline TC & $\begin{array}{c}0.24- \\
0.37\end{array}$ & $\begin{array}{c}0.66- \\
0.78\end{array}$ & $\mathbf{0 . 7 2 - 0 . 8 2}$ & $\mathbf{0 . 7 5 - 0 . 8 5}$ \\
\hline AT & - & - & $\mathbf{0 . 7 8 - 0 . 8 4}$ & $\mathbf{0 . 8 2 - 0 . 8 8}$ \\
\hline
\end{tabular}

Table 1. Segmentation results

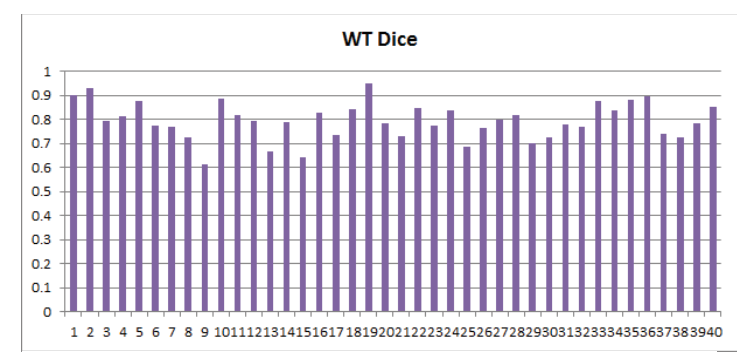

Figure 2. Dice coefficients of WT with RF

The results are shown (in Figure 2 and 3 ) for a randomly chosen 40 images from the test set having a mean of 0.793 Dice score on the whole tumor (WT) and 0.78 for the active tumor (AT) with a higher standard deviation (Figure 7 first and third boxes) . 


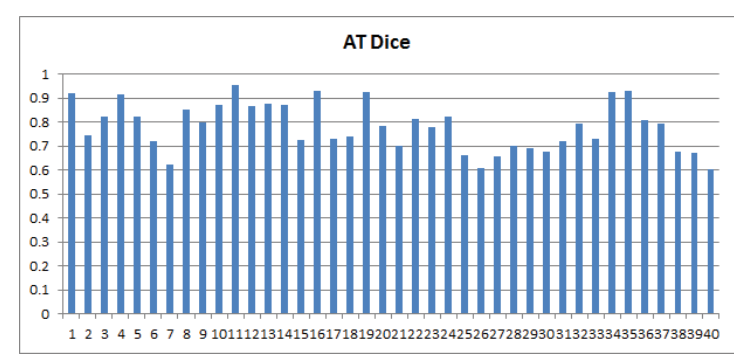

Figure 3. Dice coefficients of AT with RF

The results are also depicted graphically on a brain slice of two different images from the test set, (Figure 4). The green are the contour of the given annotation, the red are the RF segmentation results, the blue are the LSM segmentation results and the white are the ROI for LSM. We can see from these images that the delimitation surfaces between tissues are not sufficiently accurate and represent segmentation errors. It is obvious that a well-chosen local segmentation method should improve the results on the delimitation contours. Our idea was to exploit the advantages of the level set method in delimitating the borderlines of two regions belonging to two different tissues more precisely. In practice, this method may be predominantly used in the case of image zones with two tissues $\left(\Omega_{1}, \Omega_{2}\right)$ and an initial approximate delimitation surface (representing a contour in plane - C) which must be used to initialize the regions in the level set method. The specification of such regions can be done by using a mask. The level set is applied only in the image domain $(\Omega)$ delimited by the given mask.

The segmentation protocol [14] states that "various tissue elements (edema, non-enhancing, enhancing, necrosis) usually follow an outside - inside sequence" and for one tumor-tissue "it is enough to always delineate what is outside". This structure is depicted in Figure 2 - a,b containing the expert annotation (black line) in T1c and T2 modalities.

Thus, as a second stage of segmentation, after the RF segmentation, we propose to apply the level set method according to these steps:

1. The edema region looks like a homogenous and hyperintense signal in Flair images and/or low signal in T1c (Figure 4a). To improve the delimitation surface of the edema from healthy tissue, we applied the level set in a ROI (region of interest) of the Flair images. This ROI is obtained by enlarging the edema region determined in RF stage by two morphological transformations. First we created conexzone of size 3 pixels and a ball type dilatation with radius of also 3 pixels. In this way we obtained a surface $\Omega_{0}$ that includes all tumor structures in $99 \%$. The $\Omega_{0}$ is the ROI (block 7, Figure 1) where we search for the delimitation surface between the brain tissue and edema. The LSM segmentation we applied in this ROI (block 8 Figure 1) on Flair images in order to delimitate the whole tumor (WT) from the healthy tissues, being surface $\Omega_{l}$ (Figure $4 \mathrm{a}$ ).

2. We consider only the enhanced tumor, delimitated in the RF. Inside this ROI (block 13 Figure $1, \Omega=$ $\left.\Omega_{3} \cup \Omega_{4}\right)$ there are only two tissues: the enhanced tumor $\left(\Omega_{3}\right)$, which is a brightly colored tissue in the T1c modality and the necrotic core $\left(\Omega_{4}\right)$ which is dark. The level set method is able to precisely delimitate the necrotic core $\left(\Omega_{4}\right)$, in T1c modality (Figure 4d).

3. The surface of the whole tumor $\Omega_{l}$ obtained in the step 1, $\left(\Omega_{1}=\Omega_{2} \cup \Omega_{3} \cup \Omega_{4}\right)$ encapsulates all four tissues: edema with contour $\Omega_{1}$, non-enhanced tumor (contour $\Omega_{2}$ ), enhanced tumor (contour $\Omega_{3}$ ) and necrotic core (contour $\Omega_{4}$ ). The previously segmented necrotic core $\left(\Omega_{4}\right)$ has already been segmented (step 2 ) and can be eliminated from ROI. Therefore, we apply the level set only in the remaining ROI (block 11 Figure $1, \Omega=\Omega_{2}\left(\Omega_{3}\right)$ in order to find the delimitation surface of the enhanced tumor $\left(\Omega_{3}\right)$, which is brighter than the edema and non-enhanced in the T1c modality, (Figure 4b). The LSM stage delimitates the enhanced tumor surface $\Omega_{3}$ more accurately then the RF stage (block 12 Figure 1).

4. With the surface obtained from the RF segmentation stage, the whole tumor $\left(\Omega=\Omega_{1} \cup \Omega_{2} \cup \Omega_{3} \cup \Omega_{4}\right)$ encapsulates four tissues: edema $\left(\Omega_{1}\right)$, non-enhanced tumor $\left(\Omega_{2}\right)$, enhanced tumor $\left(\Omega_{3}\right)$ and necrotic core $\left(\Omega_{4}\right)$. The previously segmented zones $\left(\Omega_{3} \cup \Omega_{4}\right.$, steps 2-3) are excluded from the ROI. . So the considered ROI (block 9,10 Figure 1) contains only two tissues edema $\left(\Omega_{l}\right)$ and non-enhanced tumor $\left(\Omega_{2}\right)$. In the domain $\Omega=\Omega_{1} \cup \Omega_{2}$ we apply the LSM in order to find the delimitation surface of the non-enhanced tumor $\left(\Omega_{2}\right)$ which is slightly brighter than the edema in the T1c modality. The elimination of the enhanced tumor $\left(\Omega_{3}\right)$ before the LSM segmentation of this step ensures a more precise segmentation of the non-enhanced tumor $\left(\Omega_{2}\right)$ contour (Figure 4c)..

Applying the procedure described above, we were be able to improve our segmentation performance by 3 $7 \%$, compared to the first stage (Table 1 columns 34). The other benefit of the two-stage segmentation is the more correct delimitation of necrotic zones, to which the RF voxel-wise segmentation only offered a weak solution. Improvement brought by the second stage was measured also in terms of Dice coefficients (Table 1-column 4). Figures 5 and 6 show the numerical results referring to the same test set and measuring the Dice scores on WT and AT tumor types. 
Green contour: ground truth, red RF segmentation, white ROI for LSM, blue LSM improvement

Flair
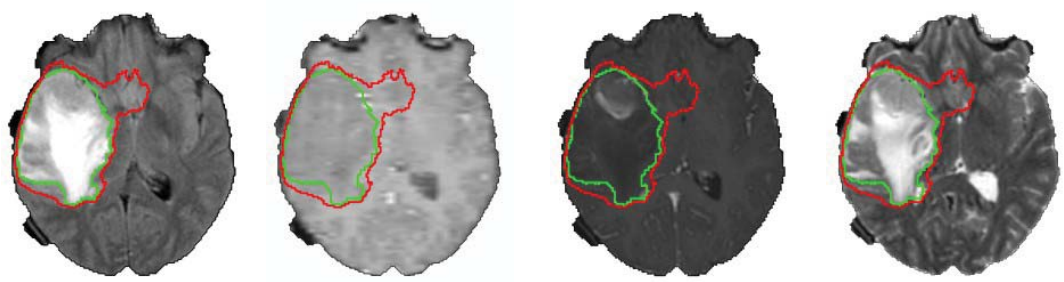

Figure 4a. Whole tumor (WT)
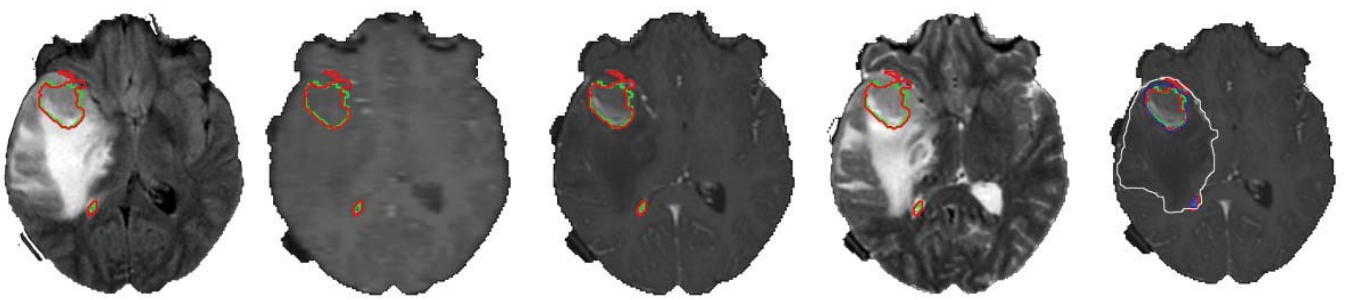

Figure $4 \mathrm{~b}$. Enhanced tumor (AT)
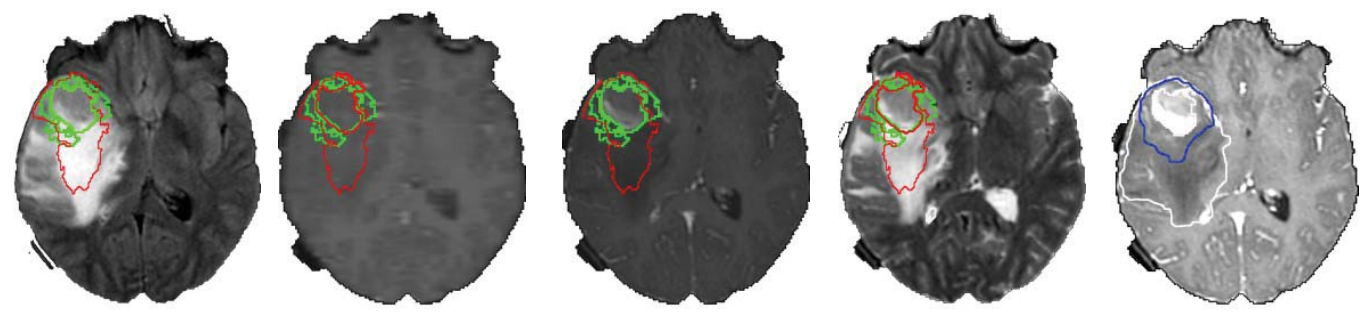

Figure 4c. Tumor core (TC)
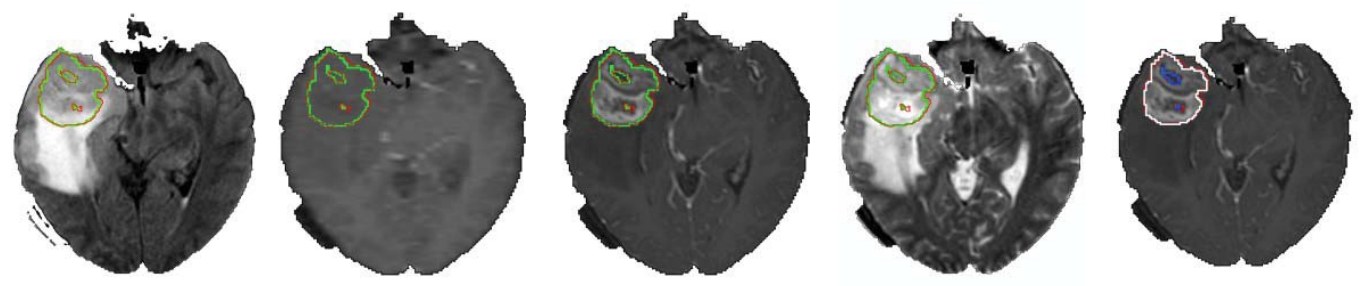

Figure 4d. Necrotic core (NC)

Figure 4. Visualized segmentation results on a brain slice

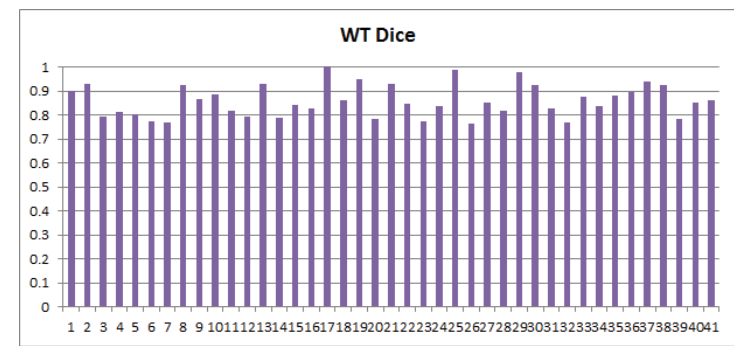

Figure 5. Dice coefficients of WT RF+LSM

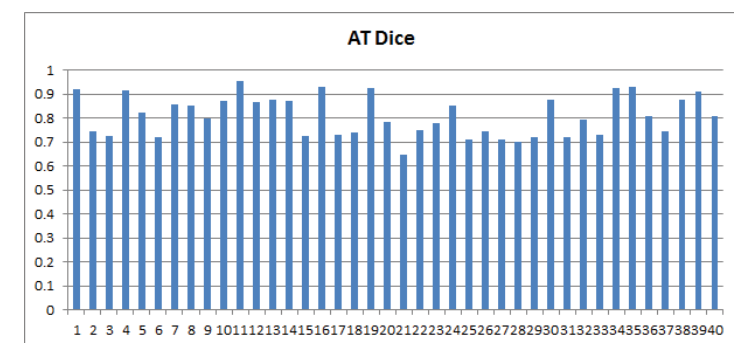

Figure 6. Dice coefficients of AT RF+LSM 


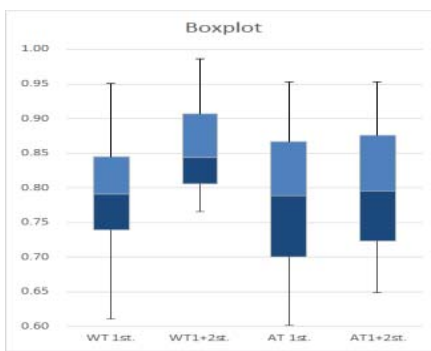

Figure 7. Boxplot comparison

The increased values are a mean of 0.854 for WT and a 0.806 for AT. These results are depicted in the boxplot also ( 2 and 4 boxes), to point out the standard deviation and the $1^{\text {st }}$ and $3^{\text {rd }}$ order quantiles (Figure 7.)

\section{CONCLUSION}

The novelty of this paper is the development of MR brain tumor segmentation framework obtained in two stages the random forest classifier linked with a welldefined sequentially applied contour refinement by the level set algorithm.

Firstly, the wise selection of features used and an adequate tuning of the random forest create a wellperforming classifier for brain tumor segmentation. Secondly, the coarse segmentation obtained by the RF approach is merged with the level set with the aim of initializing its contours. Thus, we manage to further improve the precision of delimitation surfaces between neighboring tissues. Another important benefit of the proposed approach is the better determination of the tumor tissue structure, especially that of the necrotic core inside the enhanced tumor. For the future, we propose to implement a vectorwise LSM considering all modalities simultaneously applied in 3D MRI, instead of the current contour search run consecutively in 2D slices. Finally, it should be emphasized that accurate tissue delineation is difficult even for the well-trained eye of experts, and there are significant differences between experts' opinions. Although automatic segmentation is not always tantamount to perfection, it is much faster and reproducible, providing a useful tool in computeraided medical diagnosis assistance.

\section{ACKNOWLEDGMENTS}

The work of L. Lefkovits in this article was supported by a grant of Sapientia Foundation Institute for Scientific Research (KPI), P.N. 13/19/17.05.2017. The work of S. Lefkovits was supported by UEFISCDI grant no. PN-III-P2-2.1BG-2016-0343, contract no. 114BG /01.10.2016.

\section{REFERENCES}

1. Menze BH, Jakab A, Bauer S, et al. The Multimodal Brain Tumor Image Segmentation Benchmark. IEEE Tr. Med. Imaging. 2015 34: p. 1993-2024.
2. "The SICAS Medical Image Repository". https://www.smir.ch/ BRATS/Start2015

3. Criminisi A, Shotton J. Decision forests for computer vision and medical image analysis: Springer Science \& Business Media; 2013.

4. Chang PD. Fully Convolutional Deep Residual Neural Networks for Brain Tumor Segmentation. In MICCAI-BraTS; 2016. p. 108-118.

5. Zikic D, Glocker B, Konukoglu E et al. Contextsensitive classification forests for segmentation of brain tumor tissues. In MICCAI-BraTS 2012.

6. Ellwaa A, Hussein A, Al. Naggar E et al. Brain Tumor Segmantation Using Random Forest Trained on Iteratively Selected Patients. In MICCAI-BraTS; 2016. p. 129-137.

7. Maier O, Handels H. Predicting Stroke Lesion and Clinical Outcome with Random Forests. In MICCAI-BraTS; 2016. p. 219-230.

8. Soltaninejad M, Zhang L, Lambrou $\mathrm{T}$, et al. Tumor Segmentation using Random Forests and Fully Convolutional Networks. In MICCAIBraTS; 2017 Sep. p. 279-283.

9. Simonyan K, Zisserman A. Very deep convolutional networks for large-scale image recognition. arXiv:1409.1556. 2014.

10. Chan TF, Vese LA. Active contours without edges. IEEE Transactions on image processing. 2001; 10: p. 266-277.

11 Zhao M, Lin HY, Yang CH, Hsu CY, Pan JS, Lin MJ. Automatic threshold level set model applied on MRI image segmentation of brain tissue. Applied Mathematics \& Information Sciences. 2015; 9: p. 1971-1980.

$12 \mathrm{Wu}$ YT, Chen HY, Hung CI, et al. Segmentation of Hemodynamics from Dynamic-SusceptibilityContrast Magnetic Resonance Brain Images Using Sequential Independent Component Analysis, WSCG, 2004; p. 267-274.

13. Breiman L. Random forests. Machine learning. 2001; 45: p. 5-32.

14. Jakab A. Segmenting Brain Tumors with the Slicer 3D Software Manual for providing expert segmentations for the BRATS.

15. Lefkovits L, Lefkovits S, Vaida MF. An Optimized Segmentation Framework Applied to Glioma Delimitation. Studies in Informatics and Control. 2017; 26: p. 203-212.

16. Lefkovits L, Lefkovits S, Szilágyi L. Brain Tumor Segmentation with Optimized Random Forest. In MICCAI-BraTS; 2016. p. 88-99. 